\title{
IPEC's core competency 2 roles and responsibilities: What more do we need to implement these?
}

\author{
Joann C. Harper* \\ Department of Community Health, School of Health and Human Services, National University, San Diego, CA, USA
}

Received: January 1, 2019

DOI: $10.5430 /$ jnep.v9n7p46
Accepted: February 27, 2019 Online Published: March 25, 2019

URL: https://doi.org/10.5430/jnep.v9n7p46

\begin{abstract}
The Interprofessional Education Collaborative (IPEC) has published guidelines to promote interprofessional collaboration. These guidelines are encompassed in four core competency sets. The core competencies are: Core 1: Value/Ethics, Core 2: Roles and Responsibilities, Core 3: Interprofessional Communication and Core 4: Teams and Teamwork. IPEC has outlined sub-competencies for each, which can be interpreted as a compilation of principles, behaviors, precepts and competencies. Together they serve to promote direction for interprofessional collaboration amongst health care professionals. However, the compilation may need more explanation to guide education and practice. Though the sub-competencies described in each core overlap in their application, specifically, Core 2: Roles and Responsibilities is explored for its underpinnings. The literature to date reflects educational delivery modes, but specific content is sparse, and not in the totality of the representative sub-competencies. Much of the literature omits the background that creates the context, and the content for, our deeper understanding of the principles. Therefore, important information is missing that underpins the competency statement set to teach and to learn these sub-competencies. The aim was to identify principles and applicable content to both support learning and to address barriers to learning, which may be essential to implement the sub-competency statements. The sub-competencies independent of further elucidation are unlikely to yield the comprehension needed for implementation and discernible actions that prompt interprofessional collaborative success.
\end{abstract}

Key Words: Roles and responsibilities, IPEC, Interprofessional collaboration

\section{INTRODUCTION}

The Interprofessional Education Collaborative, known as IPEC, in its sentinel publication in 2011 published " 4 " core domains with an accompanying listing of "competencies" for each domain to help guide interprofessional team practice. ${ }^{[1]}$ IPEC updated its publication in 2016 and clarified its overriding goal of interprofessional collaboration by renaming domains to core competencies as collective contributions to that goal with edits to the sub-competencies within each core. ${ }^{[2]}$ Though we might challenge whether each listing rep- resents competencies or measurable behaviors versus principles, the intent remains, and that is to help guide health care professionals to a better understanding of the codes that promote collaboration. To date IPEC stands as one of the most comprehensive and published compendia to which many US professional organizations sponsor. ${ }^{[2]}$ IPEC's Core Competency 2, Roles and Responsibilities is analyzed to identify content for, and address barriers to, its implementation and what actions may be taken to facilitate its fulfillment, consistent with interprofessional collaboration.

${ }^{*}$ Correspondence: Joann C. Harper; Email: jharper@nu.edu; Address: Department of Community Health, School of Health and Human Services, National University, San Diego, CA, USA. 
Interprofessional collaboration has been defined as the collective involvement of various professional healthcare providers working with patients, families, caregivers and communities to consider and communicate each other's perspective in delivering the highest quality of care. ${ }^{[3-5]}$ In the last few decades, the National Academy of Medicine (formerly the Institute of Medicine [IOM]) has had a profound effect to unify the goals of interprofessional collaboration in order to reduce errors affecting patients and to improve patient safety. ${ }^{[6,7]}$ The momentum continued with the IPEC publications in 2011 and 2016. ${ }^{[1,2]}$ Legislative bodies also propelled the agenda for interprofessional education ${ }^{[8]}$ forward by prompting the formation of the National Center for Interprofessional Practice and Education (NCIPE) as further evidence of the absence of adequate preparation and resources to adopt IPE as a professional imperative. ${ }^{[9]}$ While the American Association of Colleges of Nursing adopted Interprofessional (IPE) tenets in its accreditation standards prior to 2010, multiple accrediting bodies have since incorporated IPE in their accreditation standards. ${ }^{[2,10,11]}$ The multiple and recent efforts to promote IPE, and its requisites to instigate its widespread adoption, paradoxically stands to reflect the inconsistent and inadequate pace for the progress needed to adopt IPE in both academic and clinical settings.

Improved collaboration and communication have also been driven by patient-centered care, a model that emphasizes the patient at the heart of inquiry and decision-making. The prevailing goal above all others is to improve health care outcomes. IPEC's 2016 update makes it clearer that institutions, public health agencies, a cadre of providers, and the communities they serve, together create the health care team to improve outcomes of individuals and populations in several distinctly different settings. While patients is a term frequently used in the literature, individuals, clients and groups are all recipients of care from diverse providers and agencies and the terms apply to all settings.

A requisite to the goal of interprofessional collaboration is IPEC's Core Competency 2 statement: "Use the knowledge of one's own role and those of other professions to appropriately assess and address the health care needs of patients and to promote and advance the health of populations" ( $p$. 10). ${ }^{[2]}$ Each professional role varies in educational requirements, scope of practice and the legal frameworks by which each profession is allowed and required to perform. Their professional histories and evolutions are also different. Multiple teams composed of multi-functional roles are widely employed in a variety of settings, but lines get blurred, and conflicting priorities surface. These factors amongst others compel a need to delineate more explicitly the background and the content required for the understanding needed to fulfill the sub-competencies of Core Competency 2, and to overcome barriers to its implementation. See Table 1 for a listing of the sub-competencies for Core Competency 2: Roles and Responsibilities.

General Core Statement: Use the knowledge of one's own role and those of other professions to appropriately assess and address the health care needs of patients and to promote and advance the health of populations. Note: The changes from IPEC 2011 are in bold. RR is an acronym for roles and responsibilities.

Table 1. Core Competency 2: Roles and Responsibilities (RR) ${ }^{[2]}$

\begin{tabular}{|l|l|}
\hline RR1 & Communicate one's roles and responsibilities clearly to patients, families, community members, and other professionals. \\
\hline RR2 & Recognize one's limitations in skills, knowledge, and abilities. \\
\hline RR3 & $\begin{array}{l}\text { Engage diverse professionals who complement one's own professional expertise, as well as associated resources, to } \\
\text { develop strategies to meet specific health and healthcare needs of patients and populations. }\end{array}$ \\
\hline RR4 & $\begin{array}{l}\text { Explain the roles and responsibilities of other providers and how the team works together to provide care, promote health, } \\
\text { and prevent disease. }\end{array}$ \\
\hline RR5 & $\begin{array}{l}\text { Use the full scope of knowledge, skills, and abilities of professionals from health and other fields to provide care that is } \\
\text { safe, timely, efficient, effective, and equitable. }\end{array}$ \\
\hline RR6 & $\begin{array}{l}\text { Communicate with team members to clarify each member's responsibility in executing components of a treatment plan or } \\
\text { public health intervention. }\end{array}$ \\
\hline RR7 & $\begin{array}{l}\text { Forge interdependent relationships with other professions within and outside of the health system to improve care and } \\
\text { advance learning. }\end{array}$ \\
\hline RR8 & Engage in continuous professional and interprofessional development to enhance team performance and collaboration. \\
\hline RR9 & Use unique and complementary abilities of all members of the team to optimize health and patient care. \\
\hline RR10 & $\begin{array}{l}\text { Describe how professionals in health and other fields can collaborate and integrate clinical care and public health } \\
\text { interventions to optimize population health. }\end{array}$ \\
\hline
\end{tabular}




\section{BACKGROUND}

Institutions such as hospitals where a variety of disciplines reside would seemingly be the ready laboratory for practicing interprofessional collaboration, and its requisite, IPEC's Core Competency 2: Roles and responsibilities. However, these environments are stifled by hierarchical relationships, and structural frameworks that have historically inhibited collaboration. In the US up until the last few decades, most hospitals without affiliated medical schools had medical staffs comprised of independent practitioners and medical groups who had a limited allegiance to the hospital to perform to its goals. ${ }^{[12]}$ With mergers and acquisitions this has changed to create large health care systems with partnerships between physicians and hospitals, but the separate and independent roles of clinician and administrator persist. These differentiated roles are appropriate and supported by legal and regulatory mandates that require functional separation and scope of practice considerations. ${ }^{[3,13-15]}$ For instance, medical necessity determinations for care or service must be made by qualified clinicians, "physicians" without a financial interest in the decision. ${ }^{[14]}$ Though authors point to professional culture as the "means for distributing power" (p. 145), ${ }^{[16]}$ culture is also legislated by statutes, Boards of Medicine, Nursing, Pharmacy and others, accreditation standards and government, i.e., Centers for Medicare and Medicaid Services (CMS: Conditions of Participation). ${ }^{[17]}$

Further, while continuity of care has received renewed attention in the US through accountable care organizations (ACO's) and with the now transitory Affordable Care Act (ACA), hospitalist arrangements with acute hospitals have flourished. Hospitalists are physicians who care for patients in the acute setting and who generally do not have a primary or community relationship with the patient. In some cases, a hospitalist group may be caring for patients in a hospital and the contracted entity is domiciled in another state. Discontinuity and discoordination may be the outcome. Traditional roles of primary and or attending physicians have dramatically changed wherein, Hospitalists now make clinical rounds, assess and treat patients who are hospitalized. Hospitalists have replaced the role of patients' assigned primary providers or "their" pediatrician or surgeon to make rounds, which was the prevailing practice up until the early years of the 21st century. The role disruption contributes to communication disruption between primary care providers, specialists and hospitalists and between nurses, patients and their providers. ${ }^{[18,19]}$

The evolution of roles and how these affect team practice, communication and collaboration are important considerations because of the context it creates for role understanding. These factors can strike biases between professions that are unintended and even unconscious. For example, as formative and collective movements for IPE and interprofessional practice (IPP) gained modest strides, particularly within nursing and social work, in the meantime, medicine was experiencing exponential growth in specialty fields, in response to advances in technology and scientific knowledge. During this time, medicine was inward looking developing relationships between specialties. Energy was expended developing "intra-professional relations at the expense of interprofessional relations" (p. 10). ${ }^{[20]}$

To underscore the evolution premise, is another example. In the 1960's diploma, associate degree and baccalaureate degrees stood side by side as three separate programs for registered nursing license preparation. Several decades ago, the few remaining diploma programs closed. Along the way, several paths have been designed with degree offerings at various undergraduate and graduate levels, with different credentials. Advance practice nurses are now easily identified within the health care system when they hold a nurse practitioner license, but this is still not the case for clinical specialists and many other mastered-prepared nurses. Confusion is generated amongst colleagues of health-related disciplines, when mastered- or doctorate-prepared nurses are categorized as having a lesser credential than their formal preparation dictates. The lack of acknowledgement is usually unintentional. In the US, National University based in California launched the first undergraduate degree for Radiation Therapy. Additionally, a subspecialty within the Health Sciences Department has been recently developed at the graduate level for complementary and/or integrative therapies, further punctuating the importance of allied health offerings to fulfill student and public interest. Professional evolution is a reality, but it often causes temporary, frustrating and blurred role-differentiation.

Role changes may also occur that are precipitated by funding sources. These sources may be an impetus to establish imperatives for change, but can produce unique role morphologies to get something done. We can anticipate that as funding hurdles for healthcare become even more fraught with demanding choices, labor may be re-distributed and functions will change for pragmatic reasons. For instance, though the ACA promotes the relationship between hospital and community services on behalf of the patient and the continuity of care, by doing so, it disrupts traditional roles and how they function. ${ }^{[18]}$ Through decades interdisciplinary teams have functioned in hospital settings with varying degrees of success, but it is unlikely that existing teams have performed at the expected level of Core Competency 2, once again modifying self and other professions' role-perceptions. 
To grasp the development of how specific professions have evolved and continue to evolve may avoid unnecessary and disruptive assumptions counterproductive for interprofessional rapport. In one instance described, medicine's focus on sub-specialty development may have had the unintended consequences of inattention to interprofessional relationships, as a result of an unrecognized priority. In another example, nursing has spent decades to determine criteria or fundamental education or degree programs for entry into practice. The point is, health-related professions evolve and morph with the requisite education and form levels of expertise within the same profession that are often unapparent to other team members. While the specialties of medicine and surgery are widely and publicly known, this is usually not the case for the other healthcare disciplines. So, there may be role differentiation that is apparent intra-professionally, but not inter-professionally.

\section{REVIEW OF THE LITERATURE}

The literature abounds with useful and applicable learning tools, measurement instruments, and methods for the immersion of students for IPE to align with IPEC's core competencies. However, much of the literature focuses on curricula delivery, content modalities, and measurement for pre/post course performance based on attitude changes and or readiness, and does not specifically direct content to attach meaning to the words or to explicate why competencies might need to exist. ${ }^{[21-27]}$ By contrast, the literature was reviewed to discover what may be the underpinnings, constructs or definable mechanisms that support content development for the sub-competencies of IPEC's roles and responsibilities. Further, the literature from disciplines outside of the traditional health professions' cadre, were explored, with an emphasis on those factors that focus on what affects and drives human behavior and interaction within a professional context. These factors are a principal theme underlying how roles and responsibilities integrate with professional performance, and the goal of interprofessional collaboration for success.

In summary, the goal of the literature review was to identify what content may be useful to help faculty learn, and to help teach students to learn about, how professional roles are formed and how these affect their interrelationship and performance. What might we need to know in order to understand what creates role-differentiation? In addition, what might be the barriers to fulfilling the sub-competencies of roles and responsibilities? If an enriched understanding of what underpins the roles and responsibilities of professionals, and the barriers for success can be identified, IPEC's roles and responsibilities may be implemented effectively and with a better-informed approach. A deeper grasp of the barriers preventing success permits us to address these and to mitigate their influence.

\subsection{Professional culture}

Curricula may secure professional identities, but there are other influences that perpetuate the identity. Wackerhausen explains two levels of professional identity: (a) a self-image developed at the macro-level that is a blend of the profession's "official recognition" through regulations and authorizations, and the status and perception by the public it creates; and, (b) the micro-level: what it takes to possess the credentials, such as examination and practical know-how, essentially the formal qualifications. In addition, self-image is perpetuated by how one is acknowledged through the acquisition of the customs or "cultural dimensions of the profession". ${ }^{[28]}$ Accordingly, to be "one of our kind" one must behave as "our kind does" (p.459). ${ }^{[28]}$ Wackerhausen describes the "anatomy of professional identity as "talk as we do"; tell narratives with similar descriptions to reflect an experience, a patient or another's profession (p. 460). ${ }^{[28]}$ He extrapolates this idea further by positing how this affects and reinforces each profession's "understanding and valuing" of their interpretations of events, contexts, and attributes of professional identity. More importantly, professional identity precipitates an embodiment of everyday practices and habits. Why is this important? While formation of habits is an asset that keeps professionals from making serious errors, Wackerhausen argues that habituation assimilates with identity to obliterate reflection. When these habits are natural, everyday occurrences, they are embedded to escape scrutiny-these are not readily accessible or definable amongst the same or even between bordering professions. ${ }^{[28]}$ Entrenched habits formed by professional culture may become invisible barriers to communication, role differentiation, the consciousness of interdependencies, and the self-awareness needed to promote mutual respect and engagement.

\subsection{Social identity}

According to social identity theory, people classify themselves in such categories as age, gender and organizational membership. These categories also extend to prestige and power. ${ }^{[29]}$ Persons self-assign to professional, social and political groups or others assign prototypes to group members, manifested by characteristics by which persons acquire a self-identity. According to Tajfel and Turner, the essential ingredient is that individuals self-define, and are defined by others, as members of a group. We attach belongingness to ourselves as an actual or symbolic member, but whether we identify entirely with the group may be variable. ${ }^{[29]}$

Social identity theory also asserts people's sense of who 
they are, is in part, determined by their group memberships. What develops is a self-concept tied to the group and selfesteem; and therefore, a strong urge to preserve group values. According to Pecukonis, Doyle, \& Bliss, within an ethnocentrism framework “... there is an inherent sense that one's view of the world is more carefully constructed and reflective of reality than those living in other cultural groups.... In ... profession-centrism ... health professions are immersed in their individual cultures so... interacting with the world becomes an internalized template for cultural bias...." (p. 421). ${ }^{[30]}$ Many of the stereotypes ascribed to other professions are reinforced by classroom and practice environments.

\subsection{Academic environment}

Unfortunately, perceptual myths develop that are not often corrected by curricula or faculty. Because multiple health care professions take on an uni-professional curriculum at first, students grow as cohesive groups, and they develop 'in-group' alliances and others are out-groups not afforded the same trust. ${ }^{[31]}$ This is consistent with social or intergroup contact theory ${ }^{[32]}$ and social or group identity theory. ${ }^{[29,33]}$ According to Pettigrew, contact situations create contact effects. Pettigrew asserts that four interrelated processes underlie the effects of contact, which is extrapolated here to apply to other-professions and what process we may embrace: "(1)... :learning about the outgroup, (2) changing behavior, (3) generating affective ties (friendship potential) and (4) in-group reappraisal" (p.80). ${ }^{[32]}$ It puts the humanness of togetherness closer to the forefront of professional practice.

\subsection{Profession-centrism versus pro-fessionalism}

Pecukonis alleges that profession-centrism is one of the most "intractable" barriers to IPE (p. 61). ${ }^{[34]}$ It is a discipline's way of thinking and doing that is constructed. The discipline possesses its own culture by symbols, core values, curriculum content, power distribution, and determines how 'training' should proceed within the clinical setting. ${ }^{[30,34]}$ It is a parallel to ethnocentrism wherein persons attribute causes and meaning to their lives through a cultural lens, but in the IPE context, profession-centrism is applied to the health professions. The two 'centrisms' are similar in that these create insiders and outsiders by the strong group affiliation that occurs, with the creation of elitism and territorialism. ${ }^{\text {[34] }}$ However, changing the lens of the group can re-align identity for the group and for each individual too. A changed lens also has implications for how roles may be interpreted within the group to promote collaborative success.

McNair posits that professionalism is the missing ingredient in the interprofessional relationships we seek. ${ }^{[35]}$ Profession- alism and the means to describe it have undergone a number of transformations as a result of intra- and inter-professional dialogue and public opinion. However, the code now incorporates at least a focus on patients and communities as one core obligation of all health care professions. Though the standards for which she subscribes is designed for physicians, one position McNair maintains is equally applicable to all health care professions and is comprised of three principles: (a) the primacy of the patient's interest above self-interest (altruism), (b) respect for patient autonomy, and (c) social justice. Along with these principles she includes elements that are central values: accountability, excellence, duty and advocacy, service, honor and respect for others (p. 458). ${ }^{[35]} \mathrm{Her}$ position implies that uni-professionalism prevails at higher institutions as a result of our academic preparation, defined as "the pursuit of goals for single health care professional disciplines to the exclusion of other disciplines" (p. 458). ${ }^{[35]} \mathrm{It}$ therefore can be inferred that to foster inter-professionalism, a joint value system forms the basis for interprofessional practice, as she asserts. She advocates that students can learn values distinct to their discipline, but they should see their own discipline as a subgroup of a more inclusive whole of healthcare professionals. McNair's view may integrate principles into a translation for a common mission; and therefore, the footing for team dialogue. It answers the why question to form the basis to implement the core statement of Core Competency 2. See Table 1.

To continue the professionalism discussion, Englander et al. established a common taxonomy of competency domains, and did so with dual purposes, one of which was to develop a framework for a single infrastructure for curricular resources for physicians. ${ }^{[36]}$ In doing so, the authors created eight domains. They proposed these be called: "knowledge for practice". Number 5 is named "Professionalism" (p. 1092). ${ }^{[36]}$ Number 5 is included here because IPEC's 2011 and 2016 publications infer characteristics of professionalism in core and sub-competencies, but these publications do not directly address professionalism as a separate inventory. ${ }^{[1,2]}$ The professionalism domain deserves mention because of its contrast to, and the absence of, the likeness of IPEC's language in Core Competency 2. The professionalism domain excludes language that explicitly targets the roles and responsibilities, of and between, team members, though the competencies are assumed in Englander et al.'s domains 4 and 7, “interpersonal communication skills" and "interprofessional collaboration" respectively. The statements that are incorporated to describe competencies by Englander et al. may be more easily decoded to better align for measurement by comparison to IPEC's roles and responsibilities. For instance, Englander et al. use "demonstrate" as the quintessential and operative 
term as Table 2 depicts. Though "demonstrate" would need further deconstructing for measurement, to articulate behaviors that are measurable and acceptable to all health care disciplines is at least a starting point. The starting point is enabled by the capacity to assess the defining behaviors that align with the goals of competencies, such as those listed in Core Competency 2. But, more importantly, as each pro- fession delineates distinct professional competencies, these works may confound the direction IPEC provides through its sub-competencies set. Healthcare disciplines should be working from one central template to exercise interprofessional collaboration that transcends traditional boundaries of roles and responsibilities for a common goal.

Table 2. Professionalism: Demonstrate a commitment to carrying out professional responsibilities and an adherence to ethical principles ${ }^{[36]}$

\begin{tabular}{|ll|}
\hline 5.1 & Demonstrate compassion, integrity, and respect for others \\
\hline 5.2 & Demonstrate responsiveness to patient needs that supersedes self-interest \\
\hline 5.3 & Demonstrate respect for patient privacy and autonomy \\
\hline 5.4 & Demonstrate accountability to patients, society, and the profession \\
\hline 5.5 & $\begin{array}{l}\text { Demonstrate sensitivity and responsiveness to a diverse patient population, including but not limited to diversity in } \\
\text { gender, age, culture, race, religion, disabilities, and sexual orientation }\end{array}$ \\
\hline 5.6 & $\begin{array}{l}\text { Demonstrate a commitment to ethical principles pertaining to provision or withholding of care, confidentiality, informed } \\
\text { consent, and business practices, including compliance with relevant laws, policies, and regulations. }\end{array}$ \\
\hline
\end{tabular}

\section{Discussion}

\subsection{Overcoming barriers}

Peabody and Demanchick found that establishing common ground may be the first step to collaboration, ${ }^{[37]}$ but further, determining common ground may help establish a common goal as a pre-requisite to understanding roles and responsibilities. What is the purpose of the team? We sometimes believe the implicit-improving or addressing the health status of a patient or client, is explicit, when it needs to be stated clearly. From an ethical perspective, the caring and healing professions share a mission that should unite them: "to do what is best for the patient. This is their raison d'etre" (p. 457). ${ }^{[28]}$ We might unravel conflict, minimize the effects of competitive forces and renumber organizational priorities based on the most important fundamental question: What is the team's mission?

MacDonald et al. proposed that in order to use one's knowledge, but moreover, the knowledge of those of other professions, one must enact certain behaviors. They advocate for the activities in Table 3. Although item Number 6 in Table 3, "values," may be vague and intangible, it can be translated. See Table 3. ${ }^{[38]}$

Table 3. Behavioural indicators for interprofessional competency 'knowledge of the professional role of others' [38]

\begin{tabular}{|ll|}
\hline 1 & Describes where the scope of one's own profession ends and another begins. \\
\hline 2 & Openly seeks out the contributions of other team members. \\
\hline 3 & Addresses misconceptions/stereotypes among team members. \\
\hline 4 & Respects the roles, expertise, and unique contributions of other team members. \\
\hline 5 & Identifies common/overlapping professional skills amongst team members. \\
\hline 7 & Values the enhanced benefits of the collaborative efforts of the team. \\
\hline
\end{tabular}

The proposed activities in Table 3 can help us actualize roles and responsibilities provided there is a planned, active and direct effort to develop these as a result of a deeper understanding, of the whys and the barriers to, our actions. Table 3's tasks overlap considerably with IPEC's competency guidance, yet their mutual intent is congruent. But once again, too many overlapping principles or competencies emanating from several and distinct disciplines may contribute to intra- and interprofessional confusion, though they be conceptually consistent. One way of amalgamating the ideas presented is through nursing. Nursing has had a pivotal role in the plans for patient care in institutional settings; and therefore, the concept of nurses as change agents is worthy of discussion. Because teams now have a mandate to share responsibility for providing care, Orchard posits that a team plan of care that is patient-centered may replace the traditional nurse care 
plan. ${ }^{[31]}$ Similarly, Pecukonis et al. advocate for a teambased plan. ${ }^{[30]}$ Each member may assess patients or clients independently, but these independent assessments and recommendations are unified by a systematic process whereby approaches are codified for a coordinated consensus. There is a deliberate interdisciplinary development of one plan.

Co-developed plans of care may mean a dramatic shift from nurse as experts to "patient as expert of their lives" (p. 255). ${ }^{[31]}$ Orchard maintains that with interprofessional practice, nursing may fear it will be neutralized, but if nursing is able to clearly convey their role, this is unlikely. However, nursing must openly support and accept other models of practice. Nursing theories along with theories from the physical and social sciences should together enrich collaborative practice rather than promote a divided terrain in which nurse professionals insist on the exclusivity of nursing theory. Orchard challenges nurses to align with the team as collaborators versus isolationists. ${ }^{[31]}$ Nurses can encourage and lead collaboration as they are often in the key coordinator role with administrators, physicians, technologists and researchers in the day-to-day activities of institutions. They can also influence the team through behaviors that develop trust such as "keeping promises and respecting confidences" (p.4). ${ }^{[3,39]}$ These behaviors and activities can lead the way to implementing Core Competency 2.

Reflection as a practice is touted for most healthcare professions, and it is fundamental to attain self-awareness. As a practiced behavior, it has significant implications for interprofessional collaboration. Self-awareness permits the mental exercise of understanding the whys of our behaviors and shapes how we interact with others. Reflection as a professional cornerstone is taught, modeled, and practiced in a variety of ways with different degrees of intensity. Wackerhausen suggests there are productive and non-productive ways to reflect. He contends that non-reflective behavior is exhibited even during reflective exercises. ${ }^{[28]}$ Habituation may be one obstacle. Everyday practices are not necessarily topics for reflection. He considers two different kinds of reflection. The usual reflection occurs when there is a problem to be solved or ordinary circumstances become extraordinary or similar options appear without good choices. Therefore, reflection isn't practiced to challenge everyday occurrences, but when the everyday occurrence is impeded or obstructed. Wackerhausen calls this the "first order reflection".[28] This type of reflection is often molded by the profession by the determination of what gets reflected upon and the perspectives within which the reflection occurs, which are pre-dispositioned by culture and practice. First order reflection confirms, rather than challenges assumptions, identity and beliefs. He instead, proposes a "second order reflection". If we change the knowledge background and conceptual resources, new thinking and new topics will emerge. He prescribes that we visit foreign territory to cognitively immerse oneself outside of the identified profession. Interpret patterns with a different lens. ${ }^{[28]}$ Though Wackerhausen warns that destabilizing the usual modes of thinking is risky and uncomfortable, it is essential to "become a stranger to oneself" in order to question and transform a current reality (p.467). ${ }^{[28]}$ To understand the roles and thinking of another, each of us may need to change the nucleus of our scaffolds and walk within the cell of another's profession. Productive reflection is one means to support our understanding of the sub-competencies of core competency 2 , roles and responsibilities.

In summary, one way to achieve desirable behavior is to recognize the competency needed. Then, exercise the means by recognizing the scope and competencies of other disciplines as they relate to one's own. When leading or participating in teams, pursue opportunities for each professional to work at the highest end of their scope, unless proven otherwise. Team members should accept assessments from other professionals, and not duplicate them unless corroboration is desirable. Professional skills may overlap and create blurring. Professionals should identify these particular skills openly. Finally, they might embrace the support of a professional network designed for an outcome that not one individual or profession could fulfill alone. ${ }^{[38]}$

If a faculty member or a student is leading a group, a huddle or rounds, permit the time for each member to provide their version of the proposed plan of care or how their role might be exercised to achieve the desired outcome for the patient or client. ${ }^{[40]}$ Health care teams usually accomplish this already in formal meetings and with case conferences, but we should practice it in informal settings and during impromptu encounters too, so it becomes automatic. We may begin to integrate second order reflection after collegial encounters to bolster the thought process to permit thinking with a different lens and one that expands to acknowledging multiple realities.

Finally, health care professionals may incorporate practices that inform our education and enhance our understanding of others' roles. Though there is a dearth of available literature that directly informs us about what precise content supports competency development for roles and responsibilities, there are published studies that guide us about how to uncover the information that promotes these competencies. For instance, researchers embarked on a quest to identify what practicing physicians and nurses wanted to know about each other's respective roles. ${ }^{[41]}$ Focus groups comprised of physicians and nurses were engaged for a structured dialogue to reveal what they wanted to know about one another, applying IPEC's 
roles and responsibilities as a framework . One finding indicated that there was common ground between the two groups, both cited the mutual purpose of wanting to help people. Yet, the knowledge gap about roles was also recognized. The feedback indicated role understanding was challenged by the several differentiated levels of education for both professions and was often unclear, (i.e. distinguishing job responsibilities and scope at varied nurse and physician levels, for example, residents, fellows, etc. $).{ }^{[41]}$ In another study, medical and pharmacy students were paired with an advanced practice nurse (APN) to shadow the work activities of the APN to promote understanding of the APN's role. Role awareness was enhanced by students' direct observation of the responsibilities and the actions undertaken by the APN. ${ }^{[42]}$

\subsection{What else can we do?: One perspective}

There is considerable overlap in the sub-competencies listed for Core Competency 2: Roles and Responsibilities, and those also listed for Core Competency 1: Values/Ethics, Core Competency 3: Interprofessional Communication and Core Competency 4: Teams and Teamwork. One example of the overlap is, between IPEC's Core Competency 1: Values/Ethics and Core Competency 2: Roles and Responsibilities. If recognized and employed, the overlap may be useful. For instance, Core 1 may pave the way for the initial stage of team deliberation to gain a foothold about the origins of roles and responsibilities. First, Core 1 may make explicit which ethical doctrines each discipline relies upon as their framework for duty and responsibility towards patients, clients or populations. Second, it may make explicit what doctrines the team embraces to guide their function that becomes integrated in the ground rules for group discussion and decisions. What consensus can be created around these doctrines? What constitutes ethical conduct? The articulation of professional principles or values is a pre-requisite to understanding roles and responsibilities for each discipline and how each discipline perceives its professional scaffold. Finally, values and ethics should corroborate the group's all-encompassing purpose - what is the raison d'etre? If it is an altruistic one, it will have multiple translations as the group seeks to fulfill its mission, but it may need to be defined for a unified, team translation of the goal.

In curricula content, faculty should include documents that describe the legal and professional obligations of the disciplines students may encounter in their work. But as faculty, we too need to know what and how these documents are applied to practice. We might begin by sharing the doctrines, statutes and licensing requirements of our discipline to disclose what our respective accrediting bodies mandate in terms of our compliance with standards. Accreditation stan-

Published by Sciedu Press dards must be included for the program requirements of our educational institutions. But the requirements that post graduates will encounter due to the standards for which their employers are subject to, including a variety of regulatory bodies, must also be addressed. The reason is straight-forward. The basis for deliberation about roles and responsibilities begins with what each of us must do. It is likely that any one of us might be eager to forfeit a task, an assignment or a challenging heart-wrenching episode of care, but we have obligations that forbid us to do so. We may be directing care for a sub-population who may be marginalized, subject to abuse and/or living in life-threatening conditions. It may be a beginning to sort out what roles and responsibilities are by examining what cannot be negotiated or delegated and why. If we determine what the requirements for each discipline are, then we might begin the dialogue about what kinds of care and what relationships we can foster that are allowable and desirable, without legal restrictions (See Table 1:RR2 \& RR5). ${ }^{[2]}$

It takes work to build trust and rapport in a relationship. We may look inwardly and examine what does our profession mean to us as individuals? Personal identity is tied to professional identity and how well one identifies with the group builds a sense of belongingness. Failure to associate with group identity can also inhibit vital affiliations. Professionals may examine what barriers have been problematic for each of us in order to exercise our professional identity and what might prevent us from being fully committed to the team? Then, as professionals, be willing to share our perceptions and vision. To construct sustainable interdependencies between professions, we may need to recognize our fears, biases, areas of confidences, insecurities and skills and assets. It helps us convey to others our capabilities and to know what our limitations are and how much of these have been self-imposed or presumed by the perceptions of others. Where have the stereotype impressions emanated from? Professionals may need to rethink how they practice productive reflection or "second order reflection", to have a deeper sense of why we do what we do, and why others do what they do. Then, listen to the narratives of colleagues and think about their professional spheres. Most of us can start with the empathetic flank of our professions and extend it to our colleagues (See Table 1:RR7 \&RR8). ${ }^{[2]}$

Next, we may teach one another what skill sets are a part of our curricula or practice, and determine which ones are common to each of the disciplines, and which ones must be delegated to specific professionals either in a simulated or live experience. Later, as co-developed care plans or health care population plans are made, other skills will be revealed so the health care team learns together the more intricate 
levels of expertise that may be available and can be assigned. This would also permit each member to work at his or her highest level of training and experience. At the same time, it teaches each of us what may be our limitations or what others can do better. As a team we should clarify how the plan will be executed and by whom (See Table 1:RR2 \& RR6). ${ }^{[2]}$

As a team we may apply the skills to analyze a problem at hand and identify what other disciplines may be needed to address the circumstance. Team members may think in terms of how each member might complement one another, stretch their individual comfort zones, but further, what other disciplines outside of the customary health care or public health arenas should teams seek? Do the problems and their solutions represent an opportunity to partner with another entity, agency or professional group to optimize the health of an individual or population? Health care team members may enjoy the comfort and relief the professional network can bring when the answers to problems are not straight-forward (See Table 1: RR3, RR4, RR7, RR9 \& RR10). ${ }^{[2]}$

Finally, health care organizations may need to investigate the competencies associated with roles and responsibilities and what set of competencies are most compatible to the organization's mission. While many of the common disciplines represented in health care organizations, such as nursing, pharmacy, medicine and physical therapy, have accepted IPEC's core competencies through their respective accrediting bodies, not all disciplines have formally adopted these competencies. ${ }^{[2]}$ Though sub-competencies depicted within IPEC's four (4) cores do have overlapping verbiage between statements, these cores represent an integrated whole.
It may be wise for organizations to choose a set or sets of sub-competencies from one source to avoid the confusion and vagary that might arise from employing overlapping and multiple sets. The confusion may be a barrier to implementing any of the desired competencies. Instead, organizations, together with a cadre of professionals within their institutions, may consider what competencies satisfy the goals of interprofessional collaboration to promote the health and welfare of patients and populations.

\section{Conclusion}

The content outlined may be a precursor to give health care professionals the basic knowledge we need to begin the preparation of faculty and students to actualize Core Competency 2: Roles and Responsibilities. It may also overcome the initial barriers for success by generating a better understanding about what creates the barriers. The principles and constructs discussed may also establish the foundation for further development of health care professionals to enhance team performance (See Table 1:RR1 \& RR8). ${ }^{[2]}$ With more experience we may discover, and advocate for, whatever legislative changes are needed to maximize each of our roles and remove unnecessary constraints that stifle health care teams. Then, health care teams might fully effect RR5: "Use the full scope of knowledge, skills, and abilities of professionals from health and other fields to provide care that is safe, timely, efficient, effective, and equitable" (p.12). ${ }^{[2]}$

\section{CONFlicts OF INTEREST Disclosure}

The author declares there is no conflict(s) of interest.

\section{REFERENCES}

[1] Interprofessional Education Collaborative Expert Panel. Core competencies for interprofessional collaborative practice: Report of an expert panel. Washington, D.C.: Interprofessional Education Collaborative. 2011.

[2] Interprofessional Education Collaborative. Core competencies for interprofessional collaborative practice: 2016 update. Washington, DC: Interprofessional Education Collaborative. 2016.

[3] Moss, E, Seifert CP, O’Sullivan A. Registered Nurses as interprofessional collaborative partners: Creating value-based outcomes. The Online Journal of Issues in Nursing. September 30, 2016; 2(3).

[4] Sullivan M, Kiovsky RD, Mason DJ, et al. Interprofessional collaboration and education: working together to ensure excellence in health care. American Journal of Nursing. 2015; 115(3): 47-54. PMid: 25715219 https : //doi.org/10.1097/01. NAJ . 0000461 822.40440 .58

[5] World Health Organization. Framework for action on interprofessional education and collaborative practice. Geneva, Switzerland: Author. 2010.
[6] Institute of Medicine. To err is human: Building a safer health system. Washington DC: National Academy Press; 2000.

[7] Institute of Medicine. Crossing the quality chasm. Washington DC: National Academy Press; 2001.

[8] House Office of the Legislative Council. Compilation of patient protection and the Affordable Care Act Including Patient Protection and Affordable Care Act Health-Related Portions of the Health Care and Education Reconciliation Act of 2010 as Amended through May 1, 2010. 2010. Available from: http://housedocs.house.gov/ energycommerce/ppacacon.pdf

[9] National Center for Interprofessional Practice and Education. National center for interprofessional education. 2016. Available from: https://campaignforaction.org/resource/nationa 1-center-interprofessional-practice-education/

[10] American Association of Colleges of Nursing. The essentials of baccalaureate education for professional nursing practice. Report of an expert panel. 2008. Available from: https://www. aacn.nche.ed $\mathrm{u} /$ publications/order-form/baccalaureate-essentials 
[11] Accreditation Council for Pharmacy Education. Accreditation standards and key elements for the professional program in Pharmacy leading to the Doctor of Pharmacy degree. Available from: https: //www . acpe-accredit. org/pdf/Standards2016FINAL.pdf

[12] Healy JB. Principles of healthcare leadership. Washington DC.: Health Administration Press; 2018.

[13] American Nurses Association. Nursing: Scope and standards of practice. 3rd ed. Silver Spring, MD: Author. 2015.

[14] California Health \& Safety Code, Knox Keene Act of 1975, section 1340 et seq.: 2017. Available from: http://www.dmhc.ca.gov/ AbouttheDMHC/LawsRegulations . aspx\#knoxkeene

[15] The Joint Commission (n.d.). National Patient Safety Goals Effective January 2017. 2017. Retrieved from https://www. jointcommiss ion.org/assets/1/6/NPSG_Chapter_HAP_Jan2017.pdf

[16] Engum A, Jeffries PR. Collegian, interdisciplinary collisions: Bringing healthcare professionals together. SciVerse Science Direct. 2012; 19(3): 145-151.

[17] Centers for Medicare and Medicaid Services. Affordable Care Act, 2010, section 2703 (1945 of the Social Security Act). Available from: https://www.medicaid.gov/medicaid/ltss/hea lth-homes/index.html

[18] Doohan N, Devoe J. The chief primary care medical officer: Restoring continuity. Annals of Family Medicine. 2017; 15(4): 366-371. PMid:28694275 https://doi.org/10.1370/afm. 2078

[19] Gunderman R. Hospitalists and the Decline of Comprehensive Care. The New England Journal of Medicine. 2016; 375(11): 1011-1013. PMid:27509007 https://doi .org/10.1056/NEJMp1608289

[20] Barr H. Inter professional Education. Today, yesterday and tomorrow: A review. Commissioned by: The UK Centre for the Advancement of Interprofessional Education. 2005.

[21] Woltenberg L, Taylor S. Successes, challenges, and impact of a largecohort preclinical interprofessional curriculum: A four-year reflection. Currents in Pharmacy Teaching and Learning. 2018; 10(5): 662668. PMid:29986828 https://doi.org/10.1016/j.cptl.201 8.01 .003

[22] West C, Veronin M, Landry K, et al. Tools to investigate how interprofessional education activities link to competencies. Medical Education Online. 2015; 20(1): 1-4.

[23] Wang J, Zorek J. Deliberate practice as a theoretical framework for interprofessional experiential education. Frontiers in Pharmacology. 2016; 7: 188. PMid:27458378

[24] Wall C. Preparing BSN students for engagement in interprofessional learning. Nursing Education Perspectives. 2018; 39(6): 366-367. PMid:29746353 https://doi.org/10.1097/01.NEP.0000000 000000315

[25] Sevin AM, Hale KM, Brown NV, et al. Assessing interprofessional education collaborative competencies in service-learning course. American Journal of Pharmaceutical Education. 2016; 80(2): 1-8.

[26] Peeters M, Sexton M, Metz A, et al. A team-based interprofessional education course for first-year health professions students. Currents in Pharmacy Teaching and Learning. 2017; 9(6): 10991110. PMid:29233378 https://doi.org/10.1016/j.cptl.201 7.07 .006

[27] Mishoe S, Diggs L, Blando J, et al. Health professions students' teamwork before and after an interprofessional education co-curricular experience. Journal of Research in Interprofessional Practice and Education. 2018; 8(1):. 1-15.
[28] Wackerhausen S. Collaboration, professional identity and reflection across boundaries. Journal of Interprofessional Care. 2009; 23: 455473. PMid:19657938 https://doi.org/10.1080/1356182090 2921720

[29] Tajfel H, Turner J. The social identity theory of inter-group behavior. In S. Worchel \& W.G. Austin (Eds.), Psychology of Intergroup Relations. 1986.

[30] Pecukonis E, Doyle O, Bliss D. Reducing barriers to interprofessional training: Promoting interprofessional cultural competence. Journal of Interprofessional Care. 2008; 22: 417-428. PMid:18800282 https://doi.org/10.1080/13561820802190442

[31] Orchard CA. Persistent isolationist or collaborator? The nurse's role in interprofessional collaborative practice. Journal of Nursing Management. 2010; 18: 248-257. PMid:20546464 https: //doi.org/10.1111/j.1365-2834.2010.01072.x

[32] Pettigrew TF. Intergroup contact theory. Annual Review of Psychology. 1998; 49: 65-85. PMid:15012467 https://doi.org/10.114 6/annurev.psych.49.1.65

[33] Ashforth BE, Mael F. Social identity theory and the organization. The Academy of Management Review. 1989; 14(1): 20-39. https://doi.org/10.5465/amr.1989.4278999

[34] Pecukonis, E. Interprofessional education: a theoretical orientation incorporating profession-centrism and social identity theory. Journal of Law, Medicine and Ethics. 2014; 42: 60-64. PMid:25564712 https://doi.org/10.1111/jlme.12189

[35] McNair R. The case for educating healthcare students in professionalism as the core content of interprofessional education. Medical Education. 2005; 39(5): 456-464. PMid:15842679 https: //doi.org/10.1111/j.1365-2929.2005.02116.x

[36] Englander R, Cameron T, Ballard AJ, et al. Toward a common taxonomy of competency domains for the health professions and competencies for physicians. Academic Medicine. 2013; 88: 1088-1094. PMid:23807109 https://doi.org/10.1097/ACM.0b013e3182 $9 \mathrm{a} 3 \mathrm{~b} 2 \mathrm{~b}$

[37] Peabody MA, Demanchick St. P. Interprofessional opportunities: Understanding roles in collaborative practice. International Journal of Play Therapy. 2016; 25(2): 102-111. https://doi.org/10.103 7/pla0000013

[38] MacDonald MB, Bally JM, Ferguson LM, et al. Knowledge of the professional role of others: A key interprofessional competency. Nurse Education in Practice. 2010; 10(4): 238-242. PMid:20308019 https://doi.org/10.1016/j.nepr.2009.11.012

[39] Sherman RO. Boosting your influence. American Nurse Today. 2015; 10(12).

[40] Chin A, Brodie M. Ethics case: Resisting outdated models of pedagogical domination and subordination in health professions education. AMA Journal of Ethics. 2016; 18(9): 903-909. PMid:27669135 https://doi.org/10.1001/journalofethic s.2016.18.9.ecas3-1609

[41] Moriel D, Felix K, Quinlan P. Roles and responsibilities: Asking nurses and physicians what they know, do not know and want to know about the other's profession. Journal of Research in Interprofessional Practice and Education. 2017; 7(1): 1-18.

[42] Monahan L, Sparbel K, Heinschel J, et al. Medical and pharmacy students shadowing advanced practice nurses to develop interprofessional competencies. Applied Nursing Research. 2018; 39: 103-108. PMid:29422142 https://doi.org/10.1016/j .apnr. 2017.11 .012 